\title{
REDUCTION OF PLANE THERMOELASTICITY PROBLEM IN INHOMOGENEOUS STRIP TO INTEGRAL VOLTERRA TYPE EQUATION
}

\author{
YU.V. TOKOVYY ${ }^{1}$ and A.V. RYCHAHIVSKYY ${ }^{2}$ \\ ${ }^{1,2}$ Pidstryhach Institute for Applied Problems of Mechanics and Mathematics \\ Ukrainian National Academy of Sciences
}

3B Naukova, Lviv 79060, Ukraine

${ }^{2}$ Technische Universiteit Eindhoven

Den Dolech 2, Eindhoven 5600MB, The Netherlands

E-mail: ${ }^{1}$ dept11@iapmm. Iviv.ua

Received September 30, 2004; revised November 30, 2004

\begin{abstract}
We have developed a method for analytical solving of the plane thermoelasticity problem in terms of stresses for a strip, which is infinite with respect to width. To derive the governing equations, we have used a method of direct integration of differential equilibrium and compatibility equations. Reducing the governing equations to the integral Volterra type equation of the second kind, we have solved it in Fourier transforms by applying a method of simple iteration.
\end{abstract}

Key words: inhomogeneous strip, plane thermoelasticity problem

\section{Introduction}

Recently, the demands of engineering caused the development of new directions in mechanics, thermoelasticity theory of inhomogeneous solids inclusive. It is well known that all the materials are inhomogeneous to certain extent [10]. Of special interest for theoretical and practical research are the solids with continuous dependence of their elastic properties on coordinate. As an example, we mention the functionally graded materials [9], whose elastic properties can be formed technologically, composites, etc. [10].

The main methods for constructing the analytical solutions of thermoelasticity problems for inhomogeneous solids are described in $[5,7,10]$. The essential difficulty one faces while using them consists in solving the differential equations with variable coefficients. In most cases, certain approximations are used, e.g., replacement of an inhomogeneous solid by a set of conjuncted homogeneous solids [17]. 
See $[3,4,6]$ for application of such an approach to a plane elasticity problem in a strip inhomogeneous with respect to width.

Plevako showed [11] that representation of the continuously inhomogeneous material (elastic cylinder inhomogeneous in radial direction) by the soldered homogeneous layers gives a very slow convergence to an exact solution if the number of layers increases. So, he proposed to consider the inhomogeneous cylinders having such elastic characteristics that enable easy construction of the solution. Then the elastic characteristics can be approximated by continuous polylines instead of piecewiseconstant functions, improving the approximation towards an exact solution.

Despite of many approaches to solution of the thermoelasticity problems for inhomogeneous solids, there exists a strong need in analytical methods. Those methods would enable finding solutions in the form of a functional dependence on the loadings thus being efficient for different kinds of inhomogeneity, loadings, and shapes. It is known, that such solutions are most convenient for solving inverse problems of thermomechanics and the problems of optimal control of thermo-stressed state [13].

The paper deals with construction of an analytical solution of the plane thermoelasticity problem in terms of stresses for a strip inhomogeneous in its cross-section. To solve the problem, we use a method of direct integration of equilibrium and compatibility equations proposed by Vihak [14]. Such an approach enables easy application of the method for solving the problems for inhomogeneous solids, since the equilibrium equations, which are integrated directly, are independent of the mathematical model of physical relations between stresses and strains. The method has been already applied to some one-dimensional problems $[15,16]$.

\section{Statement of the Problem}

We consider a plane quasi-static thermoelasticity problem in the strip

$$
D=\{(x, y): \quad(x, y) \in(-\infty, \infty) \times[-1,1]\}
$$

for the case of inhomogeneous isotropic material. The problem is governed by the equilibrium equations $[1,8]$

$$
\left\{\begin{array}{l}
\frac{\partial \sigma_{x}}{\partial x}+\frac{\partial \sigma_{x y}}{\partial y}+F_{x}=0, \quad(x, y) \in D \\
\frac{\partial \sigma_{x y}}{\partial x}+\frac{\partial \sigma_{y}}{\partial y}+F_{y}=0
\end{array}\right.
$$

compatibility equation in terms of strains

$$
\frac{\partial^{2} e_{x}}{\partial y^{2}}+\frac{\partial^{2} e_{y}}{\partial x^{2}}=\frac{\partial^{2} e_{x y}}{\partial x \partial y}
$$

and the physical relations for plane strain $\left(e_{z}=0\right)$

$$
\begin{aligned}
& E e_{x}=\sigma_{x}-\nu\left(\sigma_{y}+\sigma_{z}\right)+\alpha E T, \quad E e_{y}=\sigma_{y}-\nu\left(\sigma_{x}+\sigma_{z}\right)+\alpha E T, \\
& \sigma_{z}=\nu\left(\sigma_{x}+\sigma_{y}\right)-\alpha E T, \quad G e_{x y}=\sigma_{x y}, \quad 2 G=E /(1+\nu) \text {. }
\end{aligned}
$$


We prescribe the tractions at the boundary

$$
\begin{array}{ll}
\left.\sigma_{y}\right|_{y=1}=-p_{1}(x), & \left.\sigma_{y}\right|_{y=-1}=-p_{2}(x), \\
\left.\sigma_{x y}\right|_{y=1}=q_{1}(x), & \left.\sigma_{x y}\right|_{y=-1}=q_{2}(x)
\end{array}
$$

and assume that stresses are tending to zero as $|x| \rightarrow \infty$. Here $\sigma_{j}, \sigma_{x y} ; e_{j}, e_{x y}$, $(j=x, y, z)$ are the stress and strain tensor components, respectively; $x=x^{*} / b$, $y=y^{*} / b ; x^{*}, y^{*}$ are the Cartesian coordinates $\left(y^{*} \in[-b, b]\right) ; E, G, \nu, \alpha$ denote the Young's modulus, shear modulus, Poisson's ratio, and the coefficient of linear thermal expansion, which are the functions of the $y$-coordinate; $F_{x}, F_{y}$ are the body forces in the dimension of stress, and $T$ denotes a prescribed temperature field. We assume that the force and thermal loadings depend on time parametrically, so, we skip the $t$-variable for shortening of notation.

\section{Reduction of the Problem to Governing Equations}

Following [14], we reduce the set of equations (2.1)-(2.4) to two governing equations for the normal stress $\sigma_{y}$ and total stress $\sigma=\sigma_{x}+\sigma_{y}$ (we call them the key stresses). To derive the first governing equation, we represent (2.2) in terms of stresses. For that, we eliminate $\sigma_{z}$ from (2.3) and express $\sigma_{x}$ in terms of the key stresses, to obtain

$$
\begin{aligned}
& 2 G e_{x}=(1-\nu) \sigma-\sigma_{y}+2 \alpha G(1+\nu) T, \\
& 2 G e_{y}=-\nu \sigma+\sigma_{y}+2 \alpha G(1+\nu) T .
\end{aligned}
$$

Using the obtained expressions for strains in terms of stresses, the fourth relation (2.3), and the equilibrium equations (2.1), we represent (2.2) in the form

$$
\begin{aligned}
\frac{\partial^{2}}{\partial y^{2}}\left(\frac{1-\nu}{2 G} \sigma+\right. & \alpha(1+\nu) T)+\frac{1-\nu}{2 G} \frac{\partial^{2} \sigma}{\partial x^{2}}+\alpha(1+\nu) \frac{\partial^{2} T}{\partial x^{2}} \\
& =\frac{\sigma_{y}}{2} \frac{d^{2}}{d y^{2}}\left(\frac{1}{G}\right)-F_{y} \frac{d}{d y}\left(\frac{1}{G}\right)-\frac{1}{2 G}\left(\frac{\partial F_{x}}{\partial x}+\frac{\partial F_{y}}{\partial y}\right) .
\end{aligned}
$$

Further, we use the relation

$$
\frac{\partial^{2} \sigma_{x}}{\partial x^{2}}+\frac{\partial F_{x}}{\partial x}=\frac{\partial^{2} \sigma_{y}}{\partial y^{2}}+\frac{\partial F_{y}}{\partial y}
$$

following from (2.1) by elimination of shear stress. Addition $\partial^{2} \sigma_{y} / \partial x^{2}$ to both sides of the latter equation yields

$$
\Delta \sigma_{y}=\frac{\partial^{2} \sigma}{\partial x^{2}}+\frac{\partial F_{x}}{\partial x}-\frac{\partial F_{y}}{\partial y}, \quad \Delta=\frac{\partial^{2}}{\partial x^{2}}+\frac{\partial^{2}}{\partial y^{2}} .
$$

We complement (3.1) - (3.2) by two boundary conditions (2.4) for $\sigma_{y}$ and those for the derivatives

$$
\left.\frac{\partial \sigma_{y}}{\partial y}\right|_{y=1}=-\frac{d q_{1}}{d x}-F_{y}(x, 1),\left.\quad \frac{\partial \sigma_{y}}{\partial y}\right|_{y=-1}=-\frac{d q_{2}}{d x}-F_{y}(x,-1) .
$$


They follow from $(2.4)_{3,4}$ by satisfying (2.1) at strip's sides $y= \pm 1$.

After determining the key stresses, the stress $\sigma_{x}$ is calculated by the formula $\sigma_{x}=\sigma-\sigma_{y}$. Finally, the shear stress is determined by integration of the equilibrium equations:

$$
\begin{aligned}
4 \sigma_{x y}=q_{1}+q_{2}- & \int_{-1}^{1}\left(\frac{\partial \sigma_{x}}{\partial x}+F_{x}\right) \\
& \operatorname{sign}(y-\xi) d \xi \\
& -\int_{-\infty}^{\infty}\left(\frac{\partial \sigma_{y}}{\partial y}+F_{y}\right) \operatorname{sign}(x-\eta) d \eta .
\end{aligned}
$$

\section{Solution of the Governing Equations}

To calculate the key stresses, we apply the integral Fourier transform [2] by $x$ to (3.1), (3.2), (2.4) 3,4 , and (3.3), to obtain the following problem in Fourier space:

$$
\begin{aligned}
& \frac{d^{2} \bar{\sigma}_{y}}{d y^{2}}-s^{2} \bar{\sigma}_{y}=-s^{2} \bar{\sigma}+i s \bar{F}_{x}-\frac{d \bar{F}_{y}}{d y} \\
& \frac{d^{2}}{d y^{2}}\left(\frac{1-\nu}{2 G} \bar{\sigma}+\alpha(1+\nu) \bar{T}\right)-s^{2}\left(\frac{1-\nu}{2 G} \bar{\sigma}+\alpha(1+\nu) \bar{T}\right) \\
& =\frac{\bar{\sigma}_{y}}{2} \frac{d^{2}}{d y^{2}}\left(\frac{1}{G}\right)-\bar{F}_{y} \frac{d}{d y}\left(\frac{1}{G}\right)-\frac{1}{2 G}\left(i s \bar{F}_{x}+\frac{d \bar{F}_{y}}{d y}\right), \\
& \left.\bar{\sigma}_{y}\right|_{y=1}=-\bar{p}_{1},\left.\quad \bar{\sigma}_{y}\right|_{y=-1}=-\bar{p}_{2}, \\
& \left.\frac{d \bar{\sigma}_{y}}{d y}\right|_{y=1}=-i s \bar{q}_{1}-\bar{F}_{y}(1),\left.\quad \frac{d \bar{\sigma}_{y}}{d y}\right|_{y=-1}=-i s \bar{q}_{2}-\bar{F}_{y}(-1) \text {. }
\end{aligned}
$$

Here $s$ denotes a parameter of the integral transform, $i=\sqrt{-1}$.

By solving $(4.1)-(4.2)$, we arrive at the expression for $\bar{\sigma}_{y}$

$$
\begin{aligned}
\bar{\sigma}_{y}= & -\bar{p}_{2} \cosh (s(1+y))-\left(i \bar{q}_{2}+\frac{1}{s} \bar{F}_{y}(-1)\right) \sinh (s(1+y)) \\
& +\int_{-1}^{y}\left(i \bar{F}_{x}(\xi)-\frac{1}{s} \frac{d \bar{F}_{y}(\xi)}{d \xi}-s \bar{\sigma}(\xi)\right) \sinh (s(y-\xi)) d \xi
\end{aligned}
$$

and two integral conditions 


$$
\begin{gathered}
\int_{-1}^{1} \bar{\sigma} \sinh (s \xi) d \xi=\left(\bar{q}_{1}+\bar{q}_{2}\right) \frac{i \sinh (s)}{s}-\left(\bar{p}_{1}-\bar{p}_{2}\right) \frac{\cosh (s)}{s} \\
\quad+\frac{\sinh (s)}{s^{2}}\left(\bar{F}_{y}(1)+\bar{F}_{y}(-1)\right)+\frac{1}{s} \int_{-1}^{1}\left(i \bar{F}_{x}-\frac{1}{s} \frac{d \bar{F}_{y}}{s}\right) \sinh (s \xi) d \xi \\
\int_{-1}^{1} \bar{\sigma} \cosh (s \xi) d \xi=\left(\bar{q}_{1}-\bar{q}_{2}\right) \frac{i \cosh (s)}{s}-\left(\bar{p}_{1}+\bar{p}_{2}\right) \frac{\sinh (s)}{s} \\
\quad+\frac{\cosh (s)}{s^{2}}\left(\bar{F}_{y}(1)-\bar{F}_{y}(-1)\right)+\frac{1}{s} \int_{-1}^{1}\left(i \bar{F}_{x}-\frac{1}{s} \frac{d \bar{F}_{y}}{d \xi}\right) \cosh (s \xi) d \xi
\end{gathered}
$$

The second equation (4.1), accompanied by (4.3), yields

$$
\begin{array}{r}
\bar{\sigma}=\frac{2 G}{1-\nu}\left\{A \cosh (s y)+B \sinh (s y)+P_{2} \bar{p}_{2}+Q_{2} \bar{q}_{2}+\Phi-\alpha(1+\nu) \bar{T}\right. \\
-\frac{1}{2} \int_{-1}^{y} \frac{d^{2}}{d \xi^{2}}\left(\frac{1}{G(\xi)}\right) \sinh (s(y-\xi)) \int_{-1}^{\xi} \bar{\sigma}(\eta) \sinh (s(\xi-\eta)) d \eta d \xi
\end{array}
$$

where

$$
\begin{aligned}
\Phi= & \frac{1}{2 s} \int_{-1}^{y} \frac{d^{2}}{d \xi^{2}}\left(\frac{1}{G(\xi)}\right) \sinh (s(y-\xi)) \int_{-1}^{\xi}\left(i \bar{F}_{x}(\eta)-\frac{1}{s} \frac{d \bar{F}_{y}(\eta)}{d \eta}\right) \\
& \times \sinh (s(\xi-\eta)) d \eta d \xi-\frac{1}{s} \int_{-1}^{y}\left[\bar{F}_{y}(\xi) \frac{d}{d \xi}\left(\frac{1}{G(\xi)}\right)\right. \\
& \left.+\frac{1}{2 G(\xi)}\left(i s \bar{F}_{x}(\xi)+\frac{d \bar{F}_{y}(\xi)}{d \xi}\right)\right] \sinh (s(y-\xi)) d \xi+\frac{Q_{2}}{i s} \bar{F}_{y}(-1), \\
P_{2}= & -\frac{1}{2 s} \int_{-1}^{y} \frac{d^{2}}{d \xi^{2}}\left(\frac{1}{G(\xi)}\right) \cosh (s(1+\xi)) \sinh (s(y-\xi)) d \xi \\
Q_{2}= & -\frac{i}{2 s} \int_{-1}^{y} \frac{d^{2}}{d \xi^{2}}\left(\frac{1}{G(\xi)}\right) \sinh (s(1+\xi)) \sinh (s(y-\xi)) d \xi
\end{aligned}
$$

The constants $A$ and $B$ are determined by (4.4).

Change of order of integration in the integral of (4.5) yields the integral Volterra type equation of the second kind for the transform of total stress: 


$$
\begin{array}{r}
\bar{\sigma}=\frac{2 G}{1-\nu}\left(A \cosh (s y)+B \sinh (s y)+P_{2} \bar{p}_{2}+Q_{2} \bar{q}_{2}+\Phi\right. \\
\left.-\alpha(1+\nu) \bar{T}-\frac{1}{2} \int_{-1}^{y} \bar{\sigma}(\eta) K(\xi, \eta, y) d \eta\right),
\end{array}
$$

where

$$
K(\xi, \eta, y)=\int_{\eta}^{y} \frac{d^{2}}{d \xi^{2}}\left(\frac{1}{G(\xi)}\right) \sinh (s(y-\xi)) \sinh (s(\xi-\eta)) d \xi
$$

Following $[15,16,18]$, we solve (4.6) by a method of simple iteration [12]:

$$
\begin{aligned}
\bar{\sigma}_{n}=\frac{2 G}{1-\nu}\left(A_{n} \cosh (s y)+B_{n}\right. & \sinh (s y)+P_{2} \bar{p}_{2}+Q_{2} \bar{q}_{2}+\Phi \\
& \left.-\alpha(1+\nu) \bar{T}-\frac{1}{2} \int_{-1}^{y} \bar{\sigma}_{n-1}(\eta) K(\xi, \eta, y) d \eta\right) .
\end{aligned}
$$

To compute the constants $A_{1}$ and $B_{1}$, it is assumed that $\bar{\sigma}_{0}=0$, and (4.4) is used.

After having found $\bar{\sigma}$, we determine $\bar{\sigma}_{y}$ by (4.3). Applying the inverse Fourier transform [2], we calculate the normal stresses $\sigma, \sigma_{y}$ and, after that, the shear stress $\sigma_{x y}$ by means of (3.4).

Note that if $\frac{1}{G}$ is linear in $y$, equation (4.6) has an exact solution already at $n=1$ :

$$
\begin{aligned}
\bar{\sigma}= & \frac{2 G}{1-\nu}\{A \cosh (s y)+B \sinh (s y)+H-\alpha(1+\nu) \bar{T}\} \\
\bar{\sigma}_{y}= & -\bar{p}_{2} \cosh (s(1+y))-\left(i \bar{q}_{2}+\frac{1}{s} \bar{F}_{y}(-1)\right) \sinh (s(1+y))-2 A s \\
& \times \int_{-1}^{y} \frac{G(\xi) \cosh (s \xi) \sinh (s(y-\xi))}{1-\nu(\xi)} d \xi-2 B s \int_{-1}^{y} \frac{G(\xi) \sinh (s \xi) \sinh (s(y-\xi))}{1-\nu(\xi)} d \xi \\
& +s \int_{-1}^{y} \frac{\alpha(\xi) E(\xi)}{1-\nu(\xi)} \bar{T}(\xi) \sinh (s(y-\xi)) d \xi-2 s \int_{-1}^{y} \frac{G(\xi) H(\xi)}{1-\nu(\xi)} \sinh (s(y-\xi)) d \xi
\end{aligned}
$$

where

$$
\begin{aligned}
A= & \frac{I_{2}}{I_{2} I_{3}-I_{1}^{2}} \Psi_{1}-\frac{I_{1}}{I_{2} I_{3}-I_{1}^{2}} \Psi_{2}, \quad B=\frac{I_{3}}{I_{2} I_{3}-I_{1}^{2}} \Psi_{2}-\frac{I_{1}}{I_{2} I_{3}-I_{1}^{2}} \Psi_{1}, \\
I_{1}= & \int_{-1}^{1} \frac{G(\xi)}{1-\nu(\xi)} \sinh (s \xi) \cosh (s \xi) d \xi, \quad I_{2}=\int_{-1}^{1} \frac{G(\xi)}{1-\nu(\xi)} \sinh ^{2}(s \xi) d \xi \\
I_{3}= & \int_{-1}^{1} \frac{G(\xi)}{1-\nu(\xi)} \cosh ^{2}(s \xi) d \xi
\end{aligned}
$$




$$
\begin{gathered}
\Psi_{1}=\frac{1}{2} \int_{-1}^{1} \bar{\sigma} \cosh (s \xi) d \xi-H_{c}+\frac{1}{2} \Theta_{c}, \quad \Psi_{2}=\frac{1}{2} \int_{-1}^{1} \bar{\sigma} \sinh (s \xi) d \xi-H_{s}+\frac{1}{2} \Theta_{s}, \\
H_{c}=\int_{-1}^{1} \frac{G(\xi) H(\xi)}{1-\nu(\xi)} \cosh (s \xi) d \xi, \quad \Theta_{c}=\int_{-1}^{1} \frac{\alpha(\xi) E(\xi) \bar{T}(\xi)}{1-\nu(\xi)} \cosh (s \xi) d \xi \\
H_{s}=\int_{-1}^{1} \frac{G(\xi) H(\xi)}{1-\nu(\xi)} \sinh (s \xi) d \xi, \quad \Theta_{s}=\int_{-1}^{1} \frac{\alpha(\xi) E(\xi) \bar{T}(\xi)}{1-\nu(\xi)} \sinh (s \xi) d \xi \\
H(y)=-\frac{1}{s} \int_{-1}^{y}\left[\bar{F}_{y}(\xi) \frac{d}{d \xi}\left(\frac{1}{G}(\xi)\right)+\frac{1}{2 G(\xi)}\left(i s \bar{F}_{x}(\xi)+\frac{d \bar{F}_{y}(\xi)}{d \xi}\right)\right] \\
\times \sinh (s(y-\xi)) d \xi
\end{gathered}
$$

and the integral expressions of $\bar{\sigma}$ are determined by (4.4).

Finally, if $E, G, \nu=$ const, then (4.7) provides us with the same expressions for $\sigma_{y}$ and $\sigma$ that have been found while solving the analogous problem for homogeneous material [14].

In the case of plane stress [1], [8], the governing equation (3.1) takes the form

$$
\begin{aligned}
\frac{\partial^{2}}{\partial y^{2}}\left(\frac{1}{E} \sigma+\alpha T\right)+ & \frac{1}{E} \frac{\partial^{2} \sigma}{\partial x^{2}}+\alpha \frac{\partial^{2} T}{\partial x^{2}} \\
& =\frac{\sigma_{y}}{2} \frac{d^{2}}{d y^{2}}\left(\frac{1}{G}\right)-F_{y} \frac{d}{d y}\left(\frac{1}{G}\right)-\frac{1}{2 G}\left(\frac{\partial F_{x}}{\partial x}+\frac{\partial F_{y}}{\partial y}\right)
\end{aligned}
$$

and (4.6) reduces to

$$
\begin{aligned}
\bar{\sigma}=E(A \cosh (s y)+B \sinh (s y)+ & P_{2} \bar{p}_{2}+Q_{2} \bar{q}_{2}+\Phi \\
& \left.-\alpha(1+\nu) \bar{T}-\frac{1}{2} \int_{-1}^{y} \bar{\sigma}(\eta) K(\xi, \eta, y) d \eta\right) .
\end{aligned}
$$

\section{Numerical Results}

Consider an inhomogeneous strip $D$ loaded by the tractions

$$
p_{1}=p_{2}=\frac{\exp \left(-x^{2}\right)}{2}, q_{1}=q_{2}=0 \text { at } F_{x}=F_{y}=T \equiv 0 \text {. }
$$

Let $G=G_{0}=$ const, $\nu=1-\frac{2}{3-a y}(a=$ const $)$. By (4.7), the Fourier transforms of $\sigma, \sigma_{y}$ are 


$$
\begin{aligned}
\bar{\sigma} & =\frac{2}{1-\nu}(A \cosh (s y)+B \sinh (s y)), \\
\bar{\sigma}_{y} & =-\bar{p}_{2} \cosh (s(1+y))-i \bar{q}_{2} \sinh (s(1+y)) \\
& -2 s A \int_{-1}^{y} \frac{\cosh (s \xi) \sinh (s(y-\xi))}{1-\nu} d \xi-2 s B \int_{-1}^{y} \frac{\sinh (s \xi) \sinh (s(y-\xi))}{1-\nu} d \xi,
\end{aligned}
$$

where $D=I_{1}^{2}-I_{2} I_{3}$

$$
\begin{aligned}
& A=\frac{1}{2 s D}\left[\left(i\left(\bar{q}_{1}+\bar{q}_{2}\right) \sinh (s)\right.\right.\left.-\left(\bar{p}_{1}-\bar{p}_{2}\right) \cosh (s)\right) I_{1} \\
&\left.+\left(i\left(\bar{q}_{2}-\bar{q}_{1}\right) \cosh (s)+\left(\bar{p}_{1}+\bar{p}_{2}\right) \sinh (s)\right) I_{2}\right], \\
& B=\frac{1}{2 s D}\left[\left(i\left(\bar{q}_{1}-\bar{q}_{2}\right) \cosh (s)-\left(\bar{p}_{1}+\bar{p}_{2}\right) \sinh (s)\right) I_{1}\right.\left.-\left(i\left(\bar{q}_{1}+\bar{q}_{2}\right) \sinh (s)-\left(\bar{p}_{1}-\bar{p}_{2}\right) \cosh (s)\right) I_{3}\right], \\
& I_{1}=\int_{-1}^{1} \frac{\sinh (s \xi) \cosh (s \xi)}{1-\nu(\xi)} d \xi, \quad I_{2}=\int_{-1}^{1} \frac{\sinh ^{2}(s \xi)}{1-\nu(\xi)} d \xi, \quad I_{3}=\int_{-1}^{1} \frac{\cosh ^{2}(s \xi)}{1-\nu(\xi)} d \xi .
\end{aligned}
$$

We see that $\bar{\sigma}, \sigma_{y}$ are independent of $G_{0}$, depending on the Poisson's ratio only, which is varying in $y$ - coordinate.

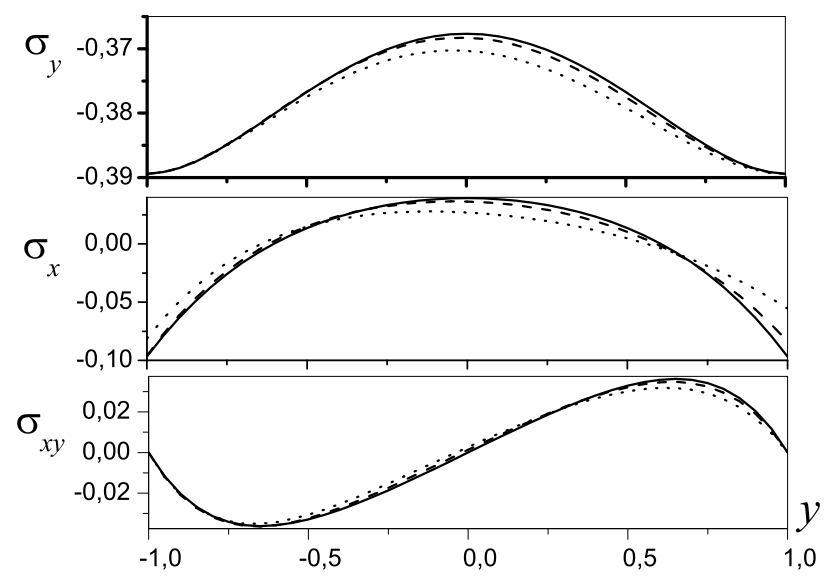

Figure 1. $y$-dependence of dimensionless stresses in $D$ at $x=0.5$ (solid line $a=0$, dashed line $-a=0.5$, dotted line $-a=1.0$ ).

Figure 1 demonstrates the $y$-distribution of dimensionless stresses in a strip $D$ for different values of the parameter $a$. The solid curves correspond to the case of 
a homogeneous material ( $a=0, \nu=0.33$ ). Then the stresses $\sigma_{y}$ and $\sigma_{x}$ are even functions of planar coordinates, while $\sigma_{x y}$ is an odd function. The dashed and dotted lines reflect the influence of material's inhomogeneity on stress distribution. Due to dependence of the Poisson's ratio on the $y$-coordinate, the law of paired normal stresses is violated. Moreover, the peaks of stresses are shifted in the direction of greater Poisson's ratio.

So, the coordinate dependence of the elastic characteristics of material has an enormous effect on the distribution of stresses.

\section{Conclusions}

The paper develops an approach to solving the plane thermoelasticity problem in terms of stresses for an inhomogeneous strip. The approach is based on the method of direct integration of differential equilibrium equations, which are independent of the mathematical model of relations between strains and stresses. Due to derived relations between stress tensor components, we can simplify calculation of the stressed state in an inhomogeneous strip considerably, if compared to solving such a problem in terms of displacements. In particular, we reduce the order of the governing differential equations with variable coefficients, derived on the basis of the compatibility and equilibrium equations.

The solution we have constructed enables calculation of the stressed state in a strip inhomogeneous with respect to width. It can be also applied for solving the corresponding inverse thermoelasticity problems as well as optimization problems.

\section{References}

[1] B. Boley and J. Weiner. Theory of Thermal Stresses. Wiley, New York, 1960.

[2] Yu. Brychkov and A. Prudnikov. Integral Transforms of Generalized Functions. Nauka, Moscow, 1977. (in Russian)

[3] V. Gorbachev. On an approach to the solution of problems in the theory of elasticity for a long anisotropic strip that is inhomogeneous with respect to width. Elasticity and Inelasticity, 39 - 54, 1993. (in Russian)

[4] V. Gorbachev and B. Pobedrya. On elastic equilibrium of inhomogeneous strips. Mekhanika tverdogo tela, 5, 111 - 118, 1979. (in Russian)

[5] G. Kolchin and E. Faverman. Theory of Elasticity of Inhomogeneous Solids. Shtiintsa, Kishinev, 1972. (in Russian)

[6] V. Lamzyuk and M. Maximova. Plane strain of a strip inhomogeneous with respect to depth. Visnyk Dnipropetrovs'koho universytetu, 2(1), 70 - 75, 1998. (in Russian)

[7] S. Lekhnitskiy. Torsion of Anisotropic Inhomogeneous Stems. Nauka, Moscow, 1971. (in Russian)

[8] R.B. Hetnarski N. Noda and Yo. Tanigava. Thermal Stresses. Lastran Corp. Rochester, New York, 2000.

[9] N. Noda. Thermal stresses in functionally graded materials. J. Thermal Stresses, 22(1), 477 - 512, 1999.

[10] V. Plevako. General Solutions in the Problems of the Theory of Elasticity of Inhomogeneous Media. Osnova, Kharkiv, 1997. (in Russian) 
[11] V. Plevako. Influence of the change of material parameters on the stressed state of an inhomogeneous cylinder. In: Proc. Intern. Scientific-Technical Conference PMM-2002, Khmelnyts'kyy, Ukraine, 2002, Problems of mathematical modeling of the modern technologies, TUP, Khmelnyts'kyy, 111, 2002. (in Ukrainian)

[12] A. Verlan' and V. Sizikov. Integral Equations: Methods, Algorithms, and Programs. Naukova Dumka, Kiev, 1988. (in Russian)

[13] V. Vigak. Control of Thermal Stresses and Displacements. Naukova Dumka, Kiev, 1988. (in Russian)

[14] V. Vihak. Direct method of integration of the equations for plane elasticity and thermoelasticity problems. Dopovidi NAN Ukrayiny, 12, 62 - 67, 1998. (in Ukrainian)

[15] V. Vihak and B. Kalynyak. Reduction of one-dimensional elasticity and thermoelasticity problems for inhomogeneous and thermo-sensitive solids to the integral equations of the second kind. Dopovidi NAN Ukrayiny, 11, 60 - 67, 1998. (in Ukrainian)

[16] V. Vihak and B. Kalynyak. Reduction of one-dimensional elasticity and thermoelasticity problems in inhomogeneous and thermal sensitive solids to the solution of integral equation of Volterra type. In: Proc. 3rd Intern. Congress 'Thermal Stresses-99', Cracow, Poland, 1999, Cracow, 457 - 460, 1999.

[17] V. Lomakin Ya. Podstrigach and Yu. Kolyano. Thermoelasticity of the Solids of Inhomogeneous Structure. Nauka, Moscow, 1984. (in Russian)

[18] A. Bondaruk Ya. Pushak and A. Rychahivskyy. Reduction of the solutions of elasticity and thermoelasticity problems for inhomogeneous solids to the integral volterra type equations. In: Proc. Intern. Scientific Conference MPMNS-2000, Lviv, Ukraine, 2000, Mathematical problems of the mechanics of inhomogeneous structures, Lviv, 315 - 318, 2000. (in Ukrainian)

\section{Nehomogeninio strypo termoelastiškumo uždavinio suvedimas į Volterra tipo integraline lygti}

Yu. Tokovyy, A. Rychahivskyy

Straipsnyje vystomas analizinio sprendinio metodas nehomogeninio strypo termoelastiškumo uždaviniui strypo ittempimams rasti, kai strypo ilgis yra begalinis pločio atžvilgiu. Pagrindinès lygtys išvedamos naudojant diferencialines pusiausvyros ir suderinamumo lygtis ir tiesiogini integravimą. Suvedus pagrindines lygtis i antrojo tipo Volterra integralinę lygti, naudojant Furje transformaciją, ji sprendžiama paprastosios iteracijos metodu. 